\title{
Hitchin Awarded Shaw Prize
}

\section{Elaine Kehoe}

The Shaw Foundation has announced the awarding of the 2016 Shaw Prize in Mathematical Sciences to NIGEL J. HITCHIN, Savilian Professor of Geometry at the University of Oxford. Hitchin was honored "for his far-reaching contributions to geometry, representation theory, and theoretical physics. The fundamental and elegant concepts and techniques that he has introduced have had wide impact and are of lasting importance."

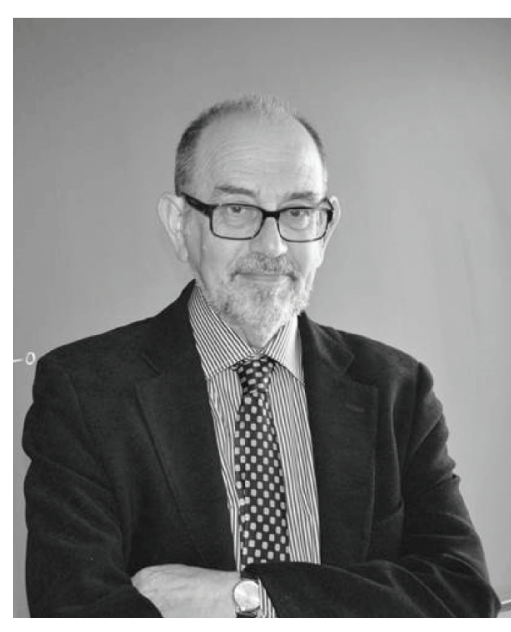

Nigel J. Hitchin his work has played a many of the spectacular interactions with theoretical physics over this period. In the 1970s, Hitchin was a leader in developing Penrose's twistor theory in the Riemannian setting and was one of the four discoverers of the ADHM construction of YangMills instantons. The part of his work that has perhaps been most influential goes back to the mid-1980s, with his introduction of Higgs bundles over Riemann surfaces, a notion that has proved to be extraordinarily fruitful and has led to major new lines of research extending into integrable systems and representation theory. But there is much more that one could mention, such as his contribution to the hyperkähler quotient construction, his invention of generalized complex structures, and his treatment of various exceptional differential-geometric phenomena through the algebra of exterior forms in low dimensions. Hitchin's work is characterized by exceptional elegance, and his novel ideas appear, in retrospect, natural and inevitable. Many of his papers fit

For permission to reprint this article, please contact:

reprint-permission@ams .org.

DOI: http://dx.doi.org/10.1090/noti1416 his new ideas into historical contexts within differential and algebraic geometry; on the other hand, new developments springing from his work are sure to continue far into the future."

\section{Biographical Sketch}

Nigel J. Hitchin was born in 1946 in Holbrook, Derbyshire, United Kingdom. He received his Diploma in Advanced Mathematics (1969) and his DPhil (1972) from Wolfson College, University of Oxford. From 1971 to 1973 he was a research assistant at the Institute for Advanced Study in Princeton, New Jersey, then became an instructor at the Courant Institute of Mathematical Sciences at New York University. He returned to Wolfson College, first as SRC Research Assistant and Junior Research Fellow (1974-1977), then as SRC Advanced Research Fellow and Research Fellow (1977-1979). From 1979 to 1990 he was Fellow and Tutor in Mathematics and CUF Lecturer at St. Catherine's College, Oxford. He became Professor of Mathematics at the University of Warwick in 1990. From 1994 to 1997 he was Rouse Ball Professor of Mathematics at the University of Cambridge and Professorial Fellow at Goville and Caius College. He has been Savilian Professor at Oxford and Professorial Fellow at New College since 1997. He has held numerous visiting positions, including at the Institut des Hautes Études Scientifiques, École Normale Supérieure, University of Bonn, the Institute for Advanced Study, and the State University of New York, Stony Brook. His awards include the Junior Whitehead Prize (1981), the Senior Berwick Prize (1990), and the Pólya Prize (2002), all from the London Mathematical Society, and the Sylvester Medal of the Royal Society of London (2000). He is a Fellow of the Royal Society and of the American Mathematical Society, and he is a member of the European Mathematical Society, the London Mathematical Society, and Academia Europea. He serves on several editorial boards, include those of the Journal of Differential Geometry and the Journal of Topology.

\section{About the Prize}

The Shaw Prize is an international award established to honor individuals who are currently active in their respective fields and who have achieved distinguished and significant advances, who have made outstanding contributions in culture and the arts, or who have achieved excellence in other domains. The award is dedicated to furthering societal progress, enhancing quality of life, and enriching humanity's spiritual civilization. Preference is 
given to individuals whose significant work was recently achieved.

The Shaw Prize consists of three annual awards: the Prize in Astronomy, the Prize in Science and Medicine, and the Prize in Mathematical Sciences. Established under the auspices of Run Run Shaw in November 2002, the prize is managed and administered by the Shaw Prize Foundation based in Hong Kong. Each prize carries a cash award of US $\$ 1,200,000$.

Previous recipients of the Shaw Prize in Mathematical Sciences are:

- Gerd Faltings and Henryk Iwaniec, 2015

- George Lusztig, 2014

- David L. Donoho, 2013

- Maxim Kontsevich, 2012

- Demetrios Christodoulou and Richard S. Hamilton, 2011

- Jean Bourgain, 2010

- Simon K. Donaldson and Clifford H. Taubes, 2009

- Vladimir Arnold and Ludwig Faddeev, 2008

- Robert Langlands and Richard Taylor, 2007

- David Mumford and Wen-Tsun Wu, 2006

- Andrew Wiles, 2005

- Shiing-Shen Chern, 2004

\section{Credit}

Photo of Nigel J. Hitchin, courtesy of Elyce Winters.

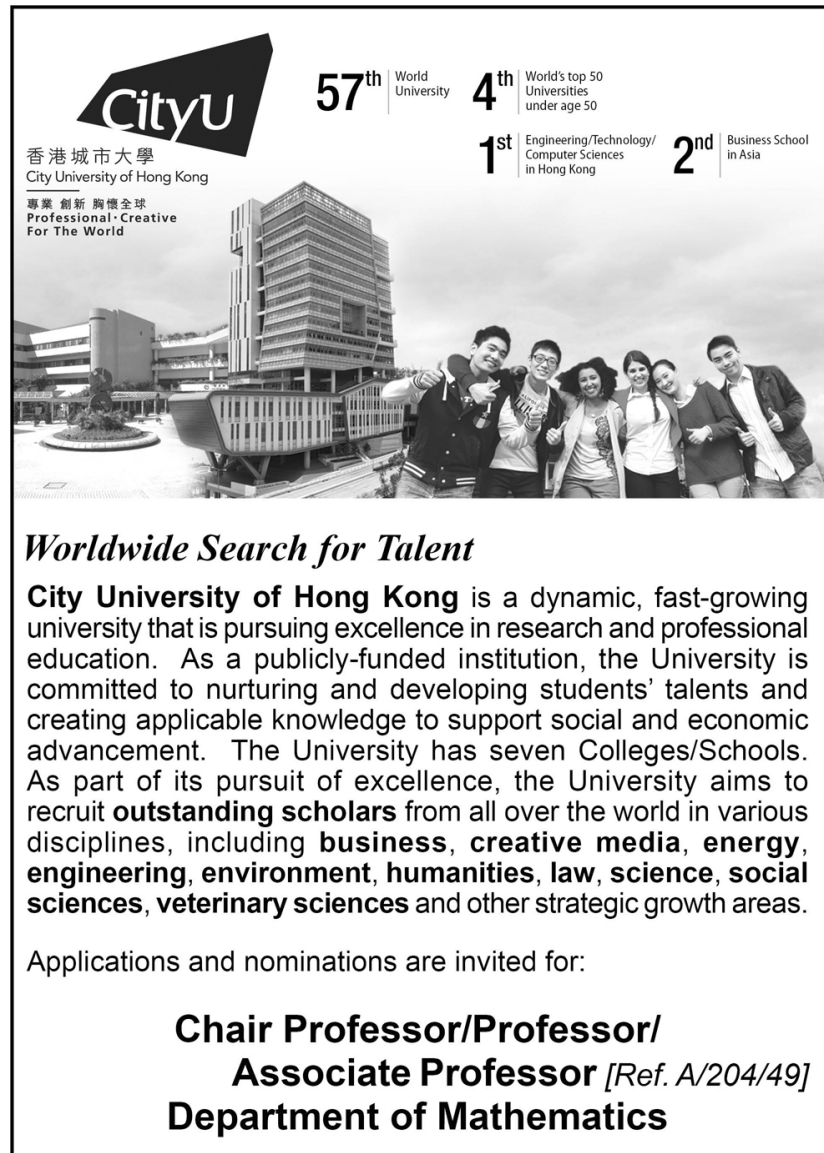

Duties : Conduct research in areas of Applied Mathematics including Analysis and Applications, Mathematical Modelling (including biological/physical/financial problems), Scientific Computation and Numerical Analysis, and Probability and Statistics; teach undergraduate and postgraduate courses; supervise research students; and perform any other duties as assigned.

Requirements : APhD in Mathematics/Applied Mathematics/ Statistics with an excellent research record.

\section{Salary and Conditions of Service}

Remuneration package will be driven by market competitiveness and individual performance. Excellent fringe benefits include gratuity, leave, medical and dental schemes, and relocation assistance (where applicable). Initial appointment will be made on a fixed-term contract.

\section{Information and Application}

Further information on the posts and the University is available at http://www.cityu.edu.hk, or from the Human Resources Office, City University of Hong Kong, Tat Chee Avenue, Kowloon Tong, Hong Kong [Email : hrojob@cityu.edu.hk/Fax : (852) 27881154 or (852) 3442 0311].

To apply, please submit an online application at http://jobs.cityu.edu.hk, and include a current curriculum vitae. Nominations can be sent directly to the Human Resources Office. Applications and nominations will receive full consideration until the positions are filled and only shortlisted applicants will be contacted. The University's privacy policy is available on the homepage.

City University of Hong Kong is an equal opportunity employer and we are committed to the principle of diversity. Personal data provided by applicants will be used for recruitment and other employment-related purposes.

Worldwide recognition ranking 57th, and 4th among top 50 universities under age 50 (QS survey 2015); 1st in Engineering/Technology/Computer Sciences in Hong Kong (Shanghai Jiao Tong University survey 2015); and 2 nd Business School in Asia-Pacific region (UT Dallas survey 2015). 